\title{
An introductory curriculum for residents on transoral robotic surgery
}

\author{
Andrea Moglia ${ }^{1}$ \\ Received: 24 February 2018 / Accepted: 3 December 2018 / Published online: 7 December 2018 \\ ๑) Springer-Verlag London Ltd., part of Springer Nature 2018
}

To the Editor,

I read with great interest the recently published article by Fastenberg et al. [1] in the Journal of Robotic Surgery on the introductory curriculum on transoral robotic surgery (TORS).

At present there is no validated simulation-based curriculum for TORS. As stated by Fastenberg et al. [1] the aim of their study is not to implement a comprehensive, but rather an introductory curriculum for TORS. It consists of completing online didactic modules and executing 12 tasks at da Vinci Skills Simulator by Intuitive Surgical (Sunnyvale, CA, USA). These exercises are the same as those assessed in a previously published study by Zhang et al. [2]. In both studies they were selected as they involve a set of skills for TORS: precision grasping, holding, wrist flexibility, and ability to cauterize vasculature. In the study by Fastenberg et al. assessment of performances at the simulator was based on the automatically generated report after completion of each task. This shows an overall score, computed after weighting several metrics which can vary among exercises but generally include: time of completion, economy of motion, instruments collisions, instruments out of view, excessive force, and master workspace range.

I have some remarks. The first is on assessment of participants. Although in this study successful completion of each simulator exercise was based on reaching a pre-determined threshold, authors did not define it. Instead, they stated that participants had to reach a large green check mark on one task before moving to the next one, without giving details on the meaning of 'large green check mark' [1].

Second, it is important to have a tutor supervising residents during simulation sessions to provide formative feedback (explanation of errors). Third, in discussion there is a subjective statement of authors for whom meaningful simulation is not possible without use of robotic surgical console. This should be supported by published evidence, e.g., on comparing performances of subjects executing the same tasks on different simulators. Currently, there are several virtual simulators available on the market [3]. These platforms enable those approaching robot-assisted surgery to become familiar with the main controls of the master console of da Vinci Surgical system, e.g., camera, clutching, and fourth arm. As such, they could be especially useful in an introductory curriculum like the one proposed by Fastenberg et al.

Acknowledgements Study supported by Fondazione Arpa (http:// www.fondazionearpa.it/).

\section{Compliance with ethical standards}

Conflict of interest Dr. Moglia declares he has no conflict of interest.

Ethical approval This article does not contain any studies with human participants or animals performed by any of the authors.

\section{References}

1. Fastenberg JH, Gibber MJ, Smith RV (2018) Introductory TORS training in an otolaryngology residency program. J Robot Surg. https://doi.org/10.1007/s11701-018-0784-7 (Epub ahead of print)

2. Zhang N, Sumer BD (2013) Transoral robotic surgery: simulationbased standardized training. JAMA Otolaryngol Head Neck Surg 139(11):1111-1117

3. Moglia A, Ferrari V, Morelli L, Ferrari M, Mosca F, Cuschieri A (2016) A Systematic review of virtual reality simulators for robot-assisted surgery. Eur Urol 69:1065-1080
Andrea Moglia

andrea.moglia@endocas.org

1 EndoCAS, Center for Computer Assisted Surgery, University of Pisa, 56124 Pisa, Italy 